\title{
QGP formation time and direct photons from heavy ion collisions
}

\author{
Fu-Ming Liu ${ }^{1}, \circledast$ and Sheng-Xu Liu ${ }^{1}$ \\ ${ }^{1}$ Key laboratory of Quark and Lepton Physics (MoE) and Institute of Particle Physics, \\ Central China Normal University, Wuhan 430079, China
}

(Dated: November 4, 2018)

\begin{abstract}
We investigated the information carried by the data of direct photons, i.e., the transverse momentum spectrum and the elliptic flow $v_{2}$ from $\mathrm{Pb}+\mathrm{Pb}$ collisions at $\sqrt{s_{N N}}=2.76 \mathrm{TeV}$ measured at Large Hadron Collider (LHC) and from $\mathrm{Au}+\mathrm{Au}$ collisions at $\sqrt{s_{N N}}=200 \mathrm{GeV}$ measured at Relativistic Heavy Ion Collider (RHIC), in the frame work of $(3+1)$-dimensional ideal hydrodynamical models constrained with hadronic data. We found those direct photon data may serve as a useful clock at the early stage of heavy ion collisions. The time scales of reaching thermal and chemical equilibrium, extracted from those data, are about $1 / 3$ and $1.5 \mathrm{fm} / \mathrm{c}$, respectively. Thus the large elliptic flow of direct photons is explainable. High order harmonics, i.e., $v_{3}, v_{4}$ and $v_{5}$, of direct photons from $\mathrm{Pb}+\mathrm{Pb}$ collisions at $2.76 \mathrm{TeV}$ are also predicted, as a further test to compete with those who claim new sources of photons to account for the large elliptic flow of direct photons.
\end{abstract}

PACS numbers:

\section{INTRODUCTION}

Recently, a large elliptic flow of direct photons, as large as that of hadrons, has been observed in heavy ion collisions, in both PHENIX experiment at RHIC and ALICE experiment at LHC [1, 2]. Such a large elliptic flow of direct photons looks puzzling because the elliptic flow of direct photons was predicted much lower than that of hadrons 3 -6].

New sources of direct photons [7, 8] have been considered to account for the large elliptic flow. They should, at one hand be constrained with the observed transverse momentum spectra of direct photons [2, 9] and hadronic data, at the other hand be tested by higher order harmonics, for example, the triangular flow $v_{3}$ of direct photons.

In this paper, we will investigate the special information carried by direct photons, after being constrained by the data of hadrons in heavy ion experiments. We will try to explained direct photon data with the delayed formation of the quark gluon plasma(QGP) in the early stage, following the some early suggestion of two time scales for thermal and chemical equilibrium $10-12$. Thus QGP formation time $\tau_{Q G P}$, the moment when the system reaches both thermal and chemical equilibrium locally, will be extracted. In order to make the calculation of direct photons constrained with hadronic data, we will take $(3+1)$-dimensional ideal hydrodynamical models [13, 14], which can give a reasonable description to hadronic data such as rapidity distribution, transverse momentum spectra, elliptic flow, etc. More than one hydro models are employed, in order to make our conclusions more general. During the whole calculation of direct photons, we will keep the solutions from those hydro models valid, and keep the equation of states consistent.

The paper is organized as following: after a brief intro-

*Electronic address: liufm@iopp.ccnu.edu.cn duction of calculate approach of direct photons in section 2 , the results will be presented in section 3 , then conclusion in section 4 .

\section{CALCULATION APPROACH}

We will consider direct photons from $\mathrm{PbPb}$ collisions at $2.76 \mathrm{TeV}$ and $\mathrm{AuAu}$ collisions at $200 \mathrm{GeV}$. The sources of direct photons are simplified as prompt photons and thermal photons, according to ALICE and PHENIX measurements of direct photons at high transverse momentum [2, 9]. Prompt photons are calculated to the nextto-leading order contribution in cold nuclear collisions:

$$
\begin{aligned}
\frac{d N^{\mathrm{P}}}{d y d^{2} p_{\mathrm{t}}} & =T_{A B}(b) \sum_{a b} \int d x_{a} d x_{b} G_{a}\left(x_{a}, M^{2}\right) G_{b}\left(x_{b}, M^{2}\right) \\
& \times \frac{\hat{s}}{\pi} \delta(\hat{s}+\hat{t}+\hat{u})\left[\frac{d \sigma}{d \hat{t}}(a b \rightarrow \gamma+X)\right. \\
& \left.+K \sum_{c} \frac{d \sigma}{d \hat{t}}(a b \rightarrow c d) \int d z_{c} \frac{1}{z_{c}^{2}} D_{\gamma / c}\left(z_{c}, Q^{2}\right)\right],
\end{aligned}
$$

where thickness function $T_{A B}(b)$, nuclear parton distribution functions $G\left(x, M^{2}\right)$ and cross sections, are the same as in previous work [15, 16]. No consideration of energy loss in fragmentation functions $D_{\gamma / c}\left(z_{c}, Q^{2}\right)$, in order to compensate the contribution from jet photon conversions. Prompt photons are supposed to carry vanishing elliptic flow.

The $p_{\mathrm{t}}$ spectrum of thermal photons reads

$$
\frac{d N^{\mathrm{T}}}{d y d^{2} p_{\mathrm{t}}}=\int d^{4} x \Gamma\left(E^{*}, T\right)
$$

where $\Gamma\left(E^{*}, T\right)$ is the photon emission rate at temperature $T$ and $E^{*}=p^{\mu} u_{\mu}, p^{\mu}$ is the four-momentum of a photon in the lab frame and $u_{\mu}$ is the flow velocity.

The hydrodynamical models [13, 14] provide us the energy density $\epsilon$ and flow velocity $u_{\mu}$ at each space-time 
point of the system for the calculation. We get the temperature at each space point according to the equation of state $\epsilon=\epsilon(T)$. We keep those solutions from hydro models valid all the time. What may be modified is the photon emission rate $\Gamma$, how strong photons are emitted.

Now we introduce the two time scales in heavy ion collisions, and discuss how photon emission depends on. The hydrodynamical description of the heavy ion systems starts from an initial time $\tau_{0}$, where a local thermal equilibrium has been assumed to solve the hydro equations. The high energy density at $\tau_{0}$ ensures a partonic phase. The ratio between quarks and gluons at $\tau_{0}$ can not be determined by hadronic data. It takes time for the system to get chemically equilibrium, thus a QGP may form at a later moment $\tau_{Q G P}$, not at $\tau_{0}$.

Quark fugacity $\xi$ is used during $\left(\tau_{0}, \tau_{Q G P}\right)$. A linear increase of quark fugacity $\xi$ from 0 at $\tau_{0}$ to unity at $\tau_{Q G P}$ is assumed in this work. The assumption of $\xi=0$ until $\tau_{0}$ is at one hand required by the large elliptic flow of direct photons, at the other hand reasonable from both CGC and EPOS initial conditions 22].

Therefore, during $\left(\tau_{0}, \tau_{Q G P}\right)$, photon emission rate is not the full rate in QGP phase $\Gamma_{A M Y}$ [17]. The Contributions from Compton process and annihilation processes, $\Gamma_{\text {Compton }}$ and $\Gamma_{\text {annihilation }}$ [18], should be modified with a factor of $\xi$ and $\xi^{2}$, respectively. Bremsstrahlung process with $n$-quark lines will be modified with a factor of $\xi^{n}$, where $n \geq 2$. It is difficult to disentangle the contribution of a given $n$-quark Bremsstrahlung. Therefore we can either ignore Bremsstrahlung contributions to get the lower limit, $\Gamma^{\text {low }}$, or overestimate them with $n=2$. Thus, during $\left(\tau_{0}, \tau_{Q G P}\right)$ photon emission rate satisfies $\Gamma^{\text {low }}<\Gamma<\Gamma^{u p}$, with the lower limit

$$
\Gamma^{\text {low }}=\xi \cdot \Gamma_{\text {Compton }}+\xi^{2} \cdot \Gamma_{\text {annihilation }},
$$

and the upper limit

$$
\Gamma^{u p}=\xi \cdot \Gamma_{\text {Compton }}+\xi^{2} \cdot\left(\Gamma_{\mathrm{AMY}}-\Gamma_{\text {Compton }}\right) .
$$

During this stage, there is also a modification of the relation $\epsilon=\epsilon(T)$ via

$$
\epsilon=\left(d_{g}+\xi d_{q}\right) \frac{\pi^{2}}{30} T^{4}
$$

where partonic degrees of freedom $d_{g}=16$ and $d_{q}=31.5$, a certain increase of the temperature, especially at very small $\xi$. But the relation between energy density and pressure remains approximately, so that hydro solutions remain valid.

Now let's summarize photon emission along the whole evolution history:

- At $\tau=0$, prompt photons are counted according to next to leading order QCD.

- During $0<\tau \leq \tau_{0}$, we have $\xi=0$ and photon emission rate $\Gamma=0$.
- During $\tau_{0}<\tau<\tau_{Q G P}$, emission will be estimated with $\Gamma^{\text {low }}<\Gamma<\Gamma^{u p}$.

- For $\tau \geq \tau_{Q G P}$, thermal photon emission rate covers both contribution from the QGP phase and hadronic phase. In QGP phase, $\Gamma_{A M Y}$ is employed. In hadronic phase, the rate is based on massive Yang-Mills (MYM) theory[19], which takes into account of both nonstrange and strangeness hadronic interactions such as $\pi+\rho \rightarrow \pi+\gamma, \pi+\pi \rightarrow \rho+\gamma$, $\pi+K^{*} \rightarrow K+\gamma$, etc.

The large elliptic flow of direct photons implies a strong emission in hadronic phase. Therefore, we don't include the dipole-like form factor in the emission rate as done in most work 15, 19]. This is not only favoured by direct photon data, but also because the form factors of those hadrons in strong interactions, or PDF of those hadrons, have not been measured. MYM theory itself remains complete without form factor.

Now we introduce how to calculate high order harmonics. The $p_{\mathrm{t}}$-spectrum of thermal photons can be decomposed into harmonics of azimuthal angle $\phi$ as

$$
\frac{d N}{d \phi} \sim 1+2 v_{2} \cos \left(\phi-\psi_{2}\right)+2 v_{3} \cos \left(\phi-\psi_{3}\right)+\ldots
$$

where $v_{2}\left(v_{n}\right)$ is the elliptic flow (higher order harmonics), and $\psi_{n}$ is the $n$-th order event plane. Obviously, $v_{n}$ and $\psi_{n}$ depend on photon's transverse momentum $p_{\mathrm{t}}$, and vary event-by-event. From eq. (6), one can easily get

$$
\begin{aligned}
& v_{n} \cos n \phi=\frac{1}{N} \int_{0}^{2 \pi} \cos n \phi \frac{d N}{d \phi} d \phi, \\
& v_{n} \sin n \phi=\frac{1}{N} \int_{0}^{2 \pi} \sin n \phi \frac{d N}{d \phi} d \phi .
\end{aligned}
$$

Let's note their right sides as $\langle\cos n \phi\rangle$ and $\langle\sin n \phi\rangle$, respectively. Then, in each event one can estimate

$$
v_{n}=\sqrt{\left.<\cos n \phi\rangle^{2}+<\sin n \phi\right\rangle^{2}},
$$

make the event average to get $v_{n}$ of thermal photons and reduce with the factor $\frac{d N^{\mathrm{T}} / d p_{t}}{d N^{\mathrm{T}} / d p_{t}+d N^{\mathrm{P}} / d p_{t}}$ to get $v_{n}$ of direct photons.

\section{RESULTS}

\section{A. An event-by-event calculation of direct photons from $\mathbf{P b}+\mathbf{P b}$ collisions at $\sqrt{s_{N N}}=2.76 \mathrm{TeV}$}

Let's start with $\mathrm{Pb}+\mathrm{Pb}$ collisions at $\sqrt{s_{N N}}=$ $2.76 \mathrm{TeV}$. Event-by-event thermal photon emission has been calculated based on EPOS2.17v3[13] where the initial time $\tau_{0}=0.35 \mathrm{fm} / \mathrm{c}$.

In Fig[1, the transverse momentum spectrum and elliptic flow $v_{2}$ of direct photons from $\mathrm{Pb}+\mathrm{Pb}$ collisions at 

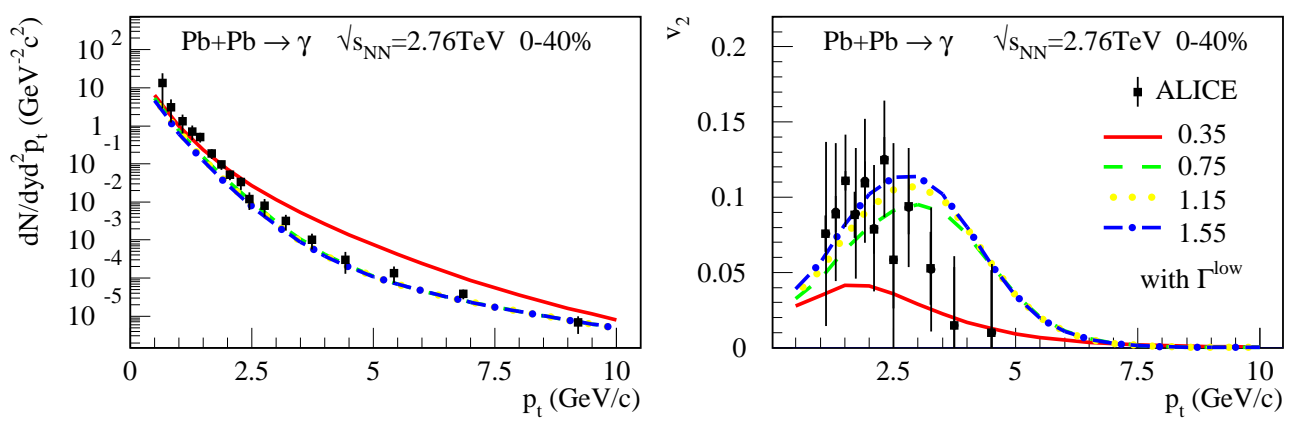

Figure 1: (Color Online) Transverse momentum spectrum and elliptic flow $v_{2}$ of direct photons from $\mathrm{Pb}+\mathrm{Pb}$ collisions at $\sqrt{s_{N N}}=2.76 \mathrm{TeV}$ for centrality $0-40 \%$, calculated with $\tau_{Q G P}=0.35,0.75,1.15$ and 1.55 fm/c. Data points from ALICE [2].

centrality $0-40 \%$ calculated with $\tau_{Q G P}=0.35,0.75,1.15$ and $1.55 \mathrm{fm} / \mathrm{c}$ are compared with the data from ALICE [2]. During $\tau_{0}<\tau<\tau_{Q G P}$, the lower emission limit was used in those curves.

Fig. 11 tells us, if QGP is formed at the initial time $\tau_{0}$, then direct photons will be overproduced, and the elliptic flow underestimated. The underestimation of elliptic flow is consistent to previous work [3 3 [6].

A delayed QGP formation will decrease the early photon emission thus increase the elliptic flow of direct photons, shown in Fig. 1. The reason is clear. Later emitted photons carry larger elliptic flow, thanks to a longer expansion of the system. Once we reduce the fraction of early emission via delayed QGP formation time, the total elliptic flow will increase.

The dependence of the transverse momentum spectrum and elliptic flow $v_{2}$ of direct photons on $\tau_{Q G P}$ can be shown more clearly in Fig. 2. The rate during $\tau_{0}<\tau<\tau_{Q G P}$ is not known explicitly, but the uncertainty is constrained with the upper (dashed lines) and lower (solid lines) limits. Here $p_{\mathrm{t}}=2.5 \mathrm{GeV} / \mathrm{c}$ are chosen to present the results, because both transverse momentum spectrum and elliptic flow show sensitivity to $\tau_{Q G P}$ here. Besides, the elliptic flow $v_{2}$ has a peak close to $2.5 \mathrm{GeV} / \mathrm{c}$.

We can see a delayed QGP formation can increase the elliptic flow of direct photons, at the same time, can decrease the transverse momentum spectrum. Now the direct photon data, extracted from ALICE data [2] and shown as full squares, are used to extract the proper QGP formation time. And a reasonable choice of $\tau_{Q G P}$ is about $1.5 \mathrm{fm} / \mathrm{c}$.

The rate during $\tau_{0}<\tau<\tau_{Q G P}$ is not known explicitly, but the uncertainty makes a theoretic error less than $10 \%$ for both the spectrum and elliptic flow of direct photons. In the following, we will not mention but use the low limit of emission rate directly.

In Fig. 3, the harmonics coefficients $v_{n}(n=2,3,4,5)$ of direct photons are predicted with $\tau_{Q G P}=1.55 \mathrm{fm} / \mathrm{c}$, accompanied with the ALICE data points of elliptic flow $v_{2}$. They behave quite similar to those of charged hadrons measured by ATLAS 20]. Thus the two-time-scale pic- ture sounds more reasonable than new sources of direct photons to account for the large elliptic flow.

\section{B. A connection between event-by-event and event-averaged calculation}

In above case, each system expands hydrodynamically based on an irregular initial condition and thermal photons are emitted event-by-event. Here we make a connection to an event-averaged calculation. The latter has an smoothed initial condition, almond-like in the transverse plane. This can be obtained from the average of the event-by-event initial conditions, with $\psi_{2}=0$ in each event, or parameterized with Glauber model. The regular system expands hydrodynamically and emits photons, similar to we did previously [6].

In Fig. 4 the elliptic flow of direct photons from averaged calculation (dashed line) is compared to event-byevent one (solid line). The same maximum of elliptic flow are obtained, no matter event-by-event or averaged calculation. But the high order harmonics such as $v_{3}, v_{4}$ and $v_{5}$ vanish in the averaged calculation because of mixing the irregular events.

The event-by-event curve moves leftward to reach the averaged curve. Further movement is needed to reach the data shape. As we know viscosity plays a more important role for irregular systems, we may attribute the deviation from data shape to the lack of viscosity in the ideal hydrodynamics.

\section{Direct photons from $\mathbf{A u}+\mathbf{A u}$ collisions at $\sqrt{s_{N N}}=200 \mathbf{G e V}$}

A correct choice of $\tau_{0}$ is also important to get the large elliptic flow. $\tau_{0}=0.35 \mathrm{fm} / \mathrm{c}$ is provided directly above. Various $\tau_{0}$ should be checked, but not available if we requires a good reproduction of hadronic data. One available case is $\tau_{0}=0.6 \mathrm{fm} / \mathrm{c}$, as our previous work 15] for $\mathrm{Au}+\mathrm{Au}$ collisions at $\sqrt{s_{N N}}=200 \mathrm{GeV}$. This is a $(3+1)$ dimensional ideal hydrodynamics 14 with Glauber initial 

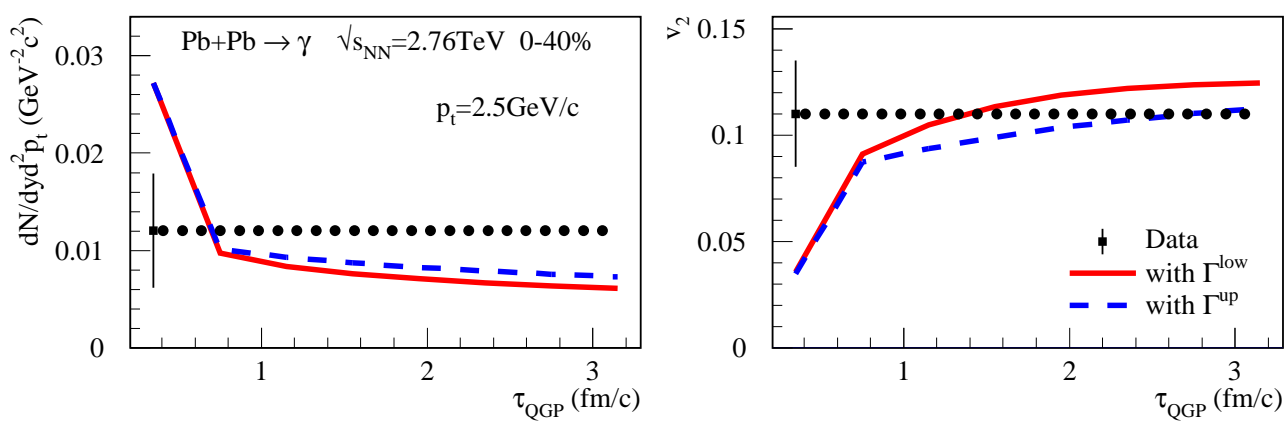

Figure 2: (Color Online) The dependence of transverse momentum spectrum and elliptic flow $v_{2}$ on $\tau_{Q G P}$ are shown at $p_{\mathrm{t}}=2.5 \mathrm{GeV}$, where dashed lines and solid lines represents calculation with up and low limits respectively between $\tau_{0}$ and $\tau_{Q G P}$. Data points are extracted from ALICE data 2] and horizontal lines are used to guide eyes.

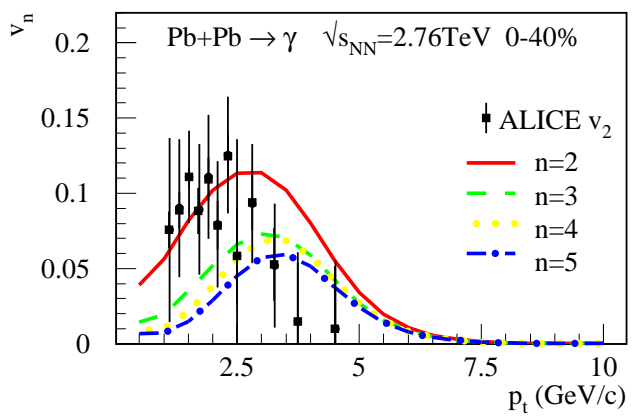

Figure 3: (Color Online) Predicted harmonics coefficients $v_{n}(n=2,3,4,5)$ of direct photons are various curves. Dots are measured $v_{2}[2]$.

condition. Averaged EPOS initial condition with $\tau_{0}=$ $0.6 \mathrm{fm} / \mathrm{c}$ provides the same elliptic flow at midrapidity [6], but a different rapidity dependence. So the following midrapidity discussion is general, valid for both of the two models.

In Fig. 5, the transverse momentum spectrum and elliptic flow $v_{2}$ of direct photons from $\mathrm{Au}+\mathrm{Au}$ collisions at $\sqrt{s_{N N}}=200 \mathrm{GeV}$ for centrality $0-20 \%$ and $20-40 \%$, calculated with $\tau_{Q G P}=0.6,1.1,1.6,2.1$ and $2.6 \mathrm{fm} / \mathrm{c}$ are compared with PHENIX data points from PHENIX [9] 1]. The previous result with hadronic form factors 15. (dashed lines) are very close to those calculated without form factors (solid lines), because the spectrum at high $p_{\mathrm{t}}$ is dominant by prompt photons, while at low $p_{\mathrm{t}}$, form factors are close to unity.

The solid curves are overlapped in the upper panels, which shows the insensitivity of spectrum to $\tau_{Q G P}$. In the lower panels, the solid curves from down to up are calculated with $\tau_{Q G P}=0.6,1.1,1.6,2.1$ and $2.6 \mathrm{fm} / \mathrm{c}$, respectively. The elliptic flow first increases with $\tau_{Q G P}$, then saturates. At $p_{\mathrm{t}}=2 \mathrm{GeV} / \mathrm{c}$, the maximum of elliptic flow is only $60 \%$ of the measured value, for both centralities. Thus, $\tau_{0}=0.6 \mathrm{fm} / \mathrm{c}$ can not work.

\section{CONCLUSION AND DISCUSSION}

The large elliptic flow and the transverse spectrum of direct photons from $\mathrm{Pb}+\mathrm{Pb}$ collisions at $\sqrt{s_{N N}}=$ $2.76 \mathrm{TeV}$ were explained with $\tau_{0} \sim 1 / 3 \mathrm{fm} / \mathrm{c}$ and $\tau_{Q G P} \sim$ $1.5 \mathrm{fm} / \mathrm{c}$. High order harmonics coefficient such as $v_{3}, v_{4}$ and $v_{5}$ of direct photons were predicted, which behave also quite similar to those variables of charged hadrons.

$\tau_{Q G P}$ has been studied systematically in this work. The test of $\tau_{0}$ has been done with two values, 0.35 and $0.6 \mathrm{fm} / \mathrm{c}$ in this work.

With $\tau_{0}=0.6 \mathrm{fm} / \mathrm{c}$, the large elliptic flow of direct photons from $\mathrm{Au}+\mathrm{Au}$ collisions at $\sqrt{s_{N N}}=200 \mathrm{GeV}$ can not be fully reproduced. The delayed QGP formation time can make the elliptic flow larger, but only up to $60 \%$ of the measured value.

More work should be done systematically to extract $\tau_{0}$ and $\tau_{Q G P}$, with systems such as AA, pA and pp. A full explanation of the data of both charged hadrons and direct photons at both colliders are expected.

\section{Acknowledgments}

This work is supported by the Natural Science Foundation of China under the project No. 11275081 and by 


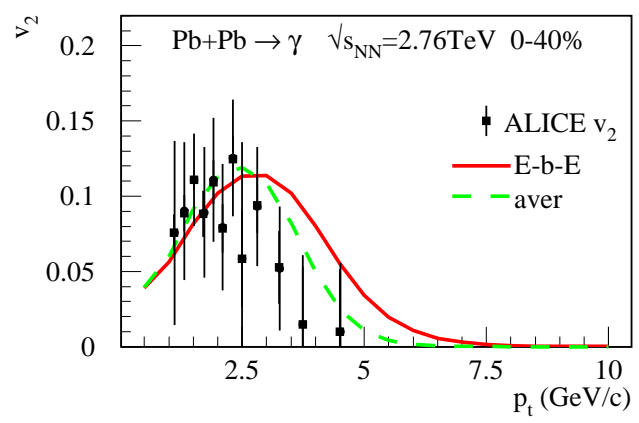

Figure 4: (Color Online) Elliptic flow of direct photons based on event-by-event and averaged calculation. Dots are measured $v_{2}[2]$.
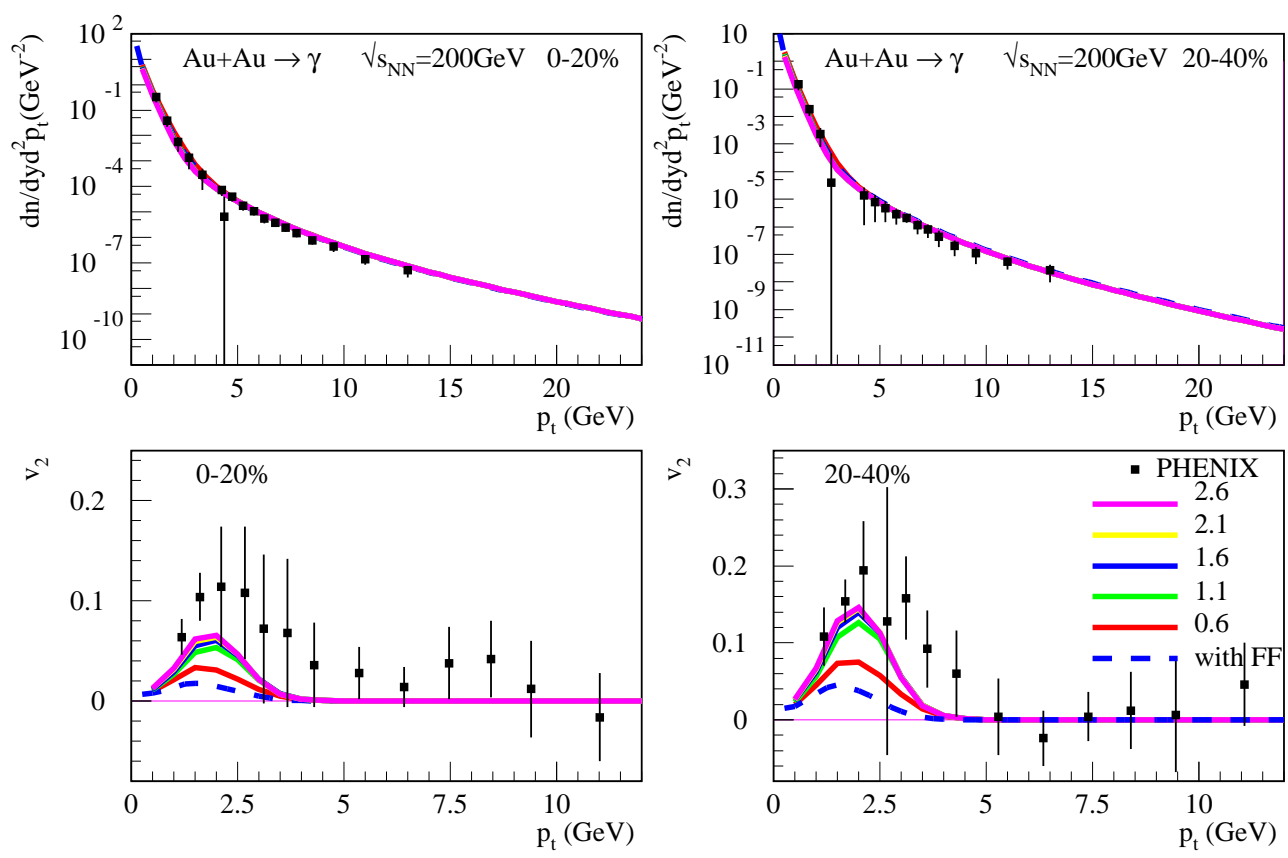

Figure 5: (Color Online) Transverse momentum spectrum and elliptic flow $v_{2}$ of direct photons from $\mathrm{Au}+\mathrm{Au}$ collisions at $\sqrt{s_{N N}}=200 \mathrm{GeV}$ for centrality $0-20 \%$ and $20-40 \%$, calculated with $\tau_{Q G P}=0.6,1.1,1.6,2.1$ and $2.6 \mathrm{fm} / \mathrm{c}$. The spectrum is not very sensitive to $\tau_{Q G P}$. The elliptic flow increases with $\tau_{Q G P}$ then saturate. Data points from PHENIX [9] [1].

Program for New Century Excellent Talents in University (NCET). FML thanks K. Werner and T. Hirano for providing the hydrodynamical evolution of two collision sys- tems, and U. Heinz, L. Mclarran, Y. Schutz, E. Shuryak and K.Werner, for very helpful discussion.
[1] A. Adare et al. [PHENIX Collaboration], Phys. Rev. Lett. 109, 122302 (2012) arXiv:1105.4126 [nucl-ex]].

[2] D. Lohner and f. t. A. Collaboration, arXiv:1212.3995 [hep-ex].

[3] H. van Hees, C. Gale and R. Rapp, Phys. Rev. C 84, 054906 (2011) arXiv:1108.2131 [hep-ph]].

[4] R. Chatterjee, H. Holopainen, I. Helenius, T. Renk and K. J. Eskola, arXiv:1305.6443 [hep-ph].

[5] R. Chatterjee, E. S. Frodermann, U. W. Heinz and
D. K. Srivastava, Phys. Rev. Lett. 96, 202302 (2006) nucl-th/0511079.

[6] F. -M. Liu, T. Hirano, K. Werner and Y. Zhu, Phys. Rev. C 80, 034905 (2009) arXiv:0902.1303 [hep-ph]].

[7] G. Basar, D. Kharzeev, D. Kharzeev and V. Skokov, Phys. Rev. Lett. 109, 202303 (2012) arXiv:1206.1334 [hep-ph]].

[8] A. Bzdak and V. Skokov, Phys. Rev. Lett. 110, 192301 (2013) arXiv:1208.5502 [hep-ph]]. 
[9] S. Afanasiev et al. [PHENIX Collaboration], Phys. Rev. Lett. 109, 152302 (2012) arXiv:1205.5759 [nucl-ex]].

[10] T. S. Biro, E. van Doorn, B. Muller, M. H. Thoma and X. N. Wang, Phys. Rev. C 48, 1275 (1993) nucl-th/9303004.

[11] E. Shuryak, Phys. Rev. Lett. 68, 3270 (1992) .

[12] E. Shuryak and L. Xiong, Phys. Rev. Lett. 70, 2241 (1993) .

[13] K. Werner, I. .Karpenko, M. Bleicher, T. Pierog and S. Porteboeuf-Houssais, Phys. Rev. C 85, 064907 (2012) arXiv:1203.5704 [nucl-th]].

[14] T. Hirano, U. Heinz, D. Kharzeev, R. Lacey, and Y. Nara, Phys. Lett. B 636, 299 (2006); J. Phys. G 34, S879 (2007); Phys. Rev. C 77, 044909 (2008).

[15] F. -M. Liu, T. Hirano, K. Werner and Y. Zhu, Phys. Rev. C 79, 014905 (2009) arXiv:0807.4771 [hep-ph]].

[16] F. -M. Liu and K. Werner, Phys. Rev. Lett. 106, 242301 (2011) arXiv:1102.1052 [hep-ph]].

[17] P. Arnold, G. D. Moore, and L. G. Yaffe, J. High Energy Phys. 0111, 057 (2001); J. High Energy Phys. 0112, 9 (2001).

[18] J. Kapusta, P. Lichard and D. Seibert, Phys. Rev. D 44:2774,(1991);47:4171(E),(1991).
[19] S. Turbide, R. Rapp and C. Gale, Phys. Rev. C 69, 014903 (2004) hep-ph/0308085.

[20] ATLAS collaboration, Phys. Rev.C86,014907, (2012).

[21] F. Gelis, E. Iancu, J. Jalilian-Marian and R. Venugopalan, Ann. Rev. Nucl. Part. Sci. 60, 463 (2010) arXiv:1002.0333 [hep-ph].

[22] It is a hot and challenging question in relativistic heavy ion physics to describe the non-equilibrium system at the early stage. The space-time evolution of quark fugacity should be determined accordingly. Here we estimate quark fugacity at $\tau_{0}$ according to initial conditions. Initial condition can be obtained from Glauber model [14], according to the distribution of nucleons in nuclei, but difficult to extract quark fugacity. Color glass condensate (CGC) model provides initial condition according to parton distribution functions [21] The small $x$ physics supports a glue-dominant system and $\xi \rightarrow 0$. Event generator EPOS 13] can provides an initial condition based on the parallel exchange of Pomerons, a kind of color tubes with vacuum quantum numbers. The longitudinal excited color tubes accommodate easily gluons, which implies $\xi \rightarrow 0$ at midrapidity. 